\title{
THE CRITICAL BEHAVIOUR OF PYROELECTRIC-TYPE SUPERCONDUCTORS
}

\author{
I.H. KRZYŻANOWSKI* \\ Laboratory of High- $\mathrm{T}_{c}$ Superconductivity, Physics Department, \\ Moscow State University, 117234 Moscow, USSR
}

(Received May 11,1990)

\begin{abstract}
The quasiclassical, Eilenberger-type equations are applied to description of rotating $P$-wave pyroelectric-type superconductor in the presence of magnetic field $\boldsymbol{H}$ and superflow $\boldsymbol{v}$. In analogy to type-II superconductors the possibility of vortex solution is discussed. We determine the symmetry of eigensolutions and the upper critical velocity $\Omega_{\mathrm{c} 2}$. We discuss the paramagnetic critical behaviour of the system depending on the strength of spin-orbit interaction.
\end{abstract}

PACS numbers: 67.50.-b, 74.70.-b

\section{Introduction}

The paper is concerned with discussion of critical properties of triplet-type (odd-parity) superfluid (superconducting) crystals without a symmetry centre. For such a system spin-orbit (S-O) interaction causes the quasiparticle energy to be spin dependent and anisotropic with respect to the direction of momentum on the Fermi surface [1-2]. The physical nature of this phenomenon is different than S-O scattering of electrons on impurities [3-4]. The discussed effect appears already in the first order of perturbation with respect to $\mathrm{S}-\mathrm{O}$ interaction.

Usually the crystal symmetry group is lowered to polar symmetry in superconductors with incommensurate charge-density-wave (as e.g. $2 \mathrm{H}-\mathrm{NbSe}_{2}$, [5]) or intercalated layered metals (since dimensions of molecules are incommensurate with the lattice period, [6]). Recently the author [7] investigated this systems and predicted the appearance of stable unhomogeneous superconducting states, which can appear within whole range of temperatures $0 \leq T \leq T_{\mathrm{c} 0}$. In the present paper

* On leave from the Institute of Physics, Technical University of Wrocław, Wybrzeże Wyspiańskiego 27, 50-370 Wrocław, Poland. 
we present the calculations of the upper critical angular velocity $\Omega_{\mathrm{c} 2}$ (for superconductors $\Omega_{\mathrm{c} 2} \sim H_{\mathrm{c} 2}$ ), as well as the Pauli limiting paramagnetic critical field. We keep the idealistic stand in which impurity scattering is ignored and the Fermi surface is spherical. Moreover, we do not explicitly include the effects of crystal field.

In Section 2 of the present paper, we apply the operator techniques [8] to transform the Gorkov equations into a set of transport-like (quasiclassical) equations.

In Section 3 we apply obtained equations to discuss the possibility of vortex solutions for rapidly rotating system (or magnetic vortex-type solution for superconductors), assuming that the system undergoes a second order phase transition at the upper critical angular velocity $\Omega_{\mathrm{c} 2}$. Assuming $\boldsymbol{\Omega} \| \hat{\boldsymbol{\varepsilon}}$-direction of polar axis we restrict ourselves to ground-like-state proportional to $\Phi\left(R_{x}, R_{y}\right)$ - the double-periodic Abrikosov's vortex function [9]. As a solution of the problem we have found that the $P$-wave states with symmetry appropriate to $Y_{21}$ and $Y_{22}$ spherical functions, exist below upper critical bound $\Omega_{\mathrm{c} 2}$. For vanishing S-O interaction influence (i.e. for non-pyroelectric crystals) the obtained states coincide with those found in superfluid ${ }^{3} \mathrm{He}[10]$. In our considerations we have assumed the pairing interaction in standard $P$-wave form. In more realistic model, however, one ought to take into account the crystal field effects onto the pairing interaction (for spin-triplet condensate [11]). Therefore, since order parameter states belonging to different irreducible representations of point symmetry of the crystal can posses different pairing interaction constants, each of the obtained states could be realized for specified pairing interaction.

In Section 4 we present analytical, as well as numerical results of the implicit equations determining $\Omega_{\mathrm{c} 2}$, as a function of temperature and reduced S-O interaction strength $e$. We demonstrate also the effects of superflow $v \| \Omega$ at $T=0$.

In Section 5 we discuss the possibility of appearance of a second order phase transition from superfluid to normal phase driven by magnetic field $\boldsymbol{H}$, acting onto the electron spins. In this Section we neglect, however, the influence of superflow $v$ and rotational frequency $\Omega$. We have constructed possible phase diagrams and predicted that depending on the symmetry of the state (with respect to $B^{J M}$ spin-tensors) and strength of S-O interaction there appear three distinct types of phase diagrams. For the first one superfluid state exists in finite (in $(e, H)$-plane) region, while for the second and third type exists in infinite region. For the second type, however, there exists region of $e$-parameter within which the system is normal for zero (and low magnitude) magnetic fields, while is driven to be superfluid for higher values of magnetic field.

\section{Formulation}

We apply the techniques developed by Eliashberg to transform the Gorkov equations, into a set of transport-like equations, which describe the behaviour of superfluid (superconducting) system. This approach was previously used for evaluation of upper critical angular velocity $\Omega_{\mathrm{c} 2}$ (upper critical magnetic field $H_{\mathrm{c} 2}$ ) 
for $P$-wave [10] and $D$-wave [12] superfluid systems. In the present paper, we consider crystals not invariant with respect to spatial inversion symmetry operation, for which the influence of spin-orbital (S-O) interaction causes the quasiparticle energy to be spin dependent $[1,2]$. For the class of pyroelectric-type systems the one-particle energy operator corresponding to a system moving with constant velocity $v$ and rotating with constant angular velocity $\Omega$ can be written in the following form:

$$
T_{\alpha \beta}(r,-\mathrm{i} \nabla)=\left[-\frac{\nabla^{2}}{2 m}-\mu+\mathrm{i}(v+\Omega \times r) \cdot \nabla\right] \delta_{\alpha \beta}+u(-\mathrm{i} \nabla) \cdot \sigma_{\alpha \beta} .
$$

Here $u(-i \nabla)$ is the anisotropic energy which appears in the first order of perturbation with respect to S-O interaction. Further we derive the Gorkov-type equations for the Matsubara-Green functions ( $G$ - normal and $F$ - anomalous) with accordance to Ref. [8]

$$
\begin{aligned}
& \left(\begin{array}{ccc}
\mathrm{i} \omega_{\nu}-\xi_{k}-v_{F} \hat{k} \cdot & \left(-\mathrm{i} \nabla_{R}-\boldsymbol{A}\right)+u(\hat{k}) \cdot \sigma & \Delta(\boldsymbol{R}, \hat{k}) \\
-\bar{\Delta}(\boldsymbol{R}, \hat{k}) & -\mathrm{i} \omega_{\nu}-\xi_{k}-v_{F} \hat{k} \cdot\left(-\mathrm{i} \nabla_{R}+\boldsymbol{A}\right)+u(\hat{k}) \cdot \sigma^{t r}
\end{array}\right) \\
& \times\left(\begin{array}{cc}
G\left(\boldsymbol{R}, k ; \mathrm{i} \omega_{\nu}\right) & F\left(\boldsymbol{R}, k ; \mathrm{i} \omega_{\nu}\right) \\
-\bar{F}\left(\boldsymbol{R}, k ; \mathrm{i} \omega_{\nu}\right) & -\bar{G}\left(\boldsymbol{R}, k ; \mathrm{i} \omega_{\nu}\right)
\end{array}\right)=\hat{1},
\end{aligned}
$$

where we have introduced the following abbreviations:

$$
\begin{aligned}
\xi_{k} & =\left(k^{2} / 2 m-\mu\right) \approx k_{F} / m\left(|k|-k_{F}\right)=v_{F}\left(|k|-k_{F}\right), \\
\boldsymbol{A} & =m(v+\Omega \times r) .
\end{aligned}
$$

For $u$ function we take only the first term of expansion with respect to spherical functions, i.e. $u(\hat{k})=\hat{\varepsilon} \times \hat{k}$ ( $k$-momentum is chosen to lie on the Fermi surface and $\hat{\varepsilon}$ is the direction of the polar axis, [2]). In the above equation $\Delta$ denotes the matrix order parameter, determined by the following gap equation:

$$
\Delta_{\alpha \beta}(\boldsymbol{R}, \hat{k})=T \sum_{\omega_{\nu}} \int \frac{\mathrm{d}^{3} k_{1}}{(2 \pi)^{3}} g\left(k-k_{1}\right) F_{\alpha \beta}\left(\boldsymbol{R}, k_{1} ; i \omega_{\nu}\right) .
$$

In applied notation $\boldsymbol{R}$ denotes the centre-of-mass coordinate of the quasiparticle pair and $k$ its momentum. Let us note that the above differential equations are derived in the framework of weak coupling approximation and additionally their validity is confined to $|p| \ll|k| \approx k_{F}$ region ( $p$ is the centre-of-mass momentum corresponding to the operator $-\mathrm{i} \nabla_{R}$ ). Making use of BCS approximation

$$
g\left(k-k_{1}\right)=\left\{\begin{array}{lll}
g\left(k \cdot k_{1}\right), & \left|\xi_{k}\right|<\omega_{c}, & \left|\xi_{k 1}\right|<\omega_{c}, \\
0, & \text { otherwise }
\end{array}\right.
$$

and employing the expansion in terms of Legendre polynomials $P_{\ell}\left(\hat{k} \cdot \hat{k}^{\prime}\right)$, i.e.

$$
g\left(\hat{k} \cdot \hat{k}^{\prime}\right)=\sum_{l=0}^{+\infty}(2 l+1) g_{\ell} P_{\ell}\left(\hat{k} \cdot \hat{k}^{\prime}\right)
$$


we obtain the gap equation (with $N(0)$ - the density of states on the Fermi surface):

$$
\Delta_{\alpha \beta}(\boldsymbol{R}, \hat{k})=T \sum_{l=0}^{+\infty}(2 l+1) g_{\ell} N(0) \sum_{\left|\omega_{\nu}\right|<\omega_{c}} \int \mathrm{d} \xi \int \frac{\mathrm{d} \Omega_{1}}{4 \pi} P_{\ell}\left(k \cdot k_{1}\right) F_{\alpha \beta}\left(\boldsymbol{R}, k_{1} ; \mathrm{i} \omega_{\nu}\right) .(7)
$$

Since for description of superfluid system one does not need all the information contained in Green functions, following Eilenberger [8], after some operator transformation, we write a set of transport-like equations, which enables one to determine the $\xi$-integrated Green functions:

$$
\begin{aligned}
& \left(2 i \omega_{\nu}+K_{-}\right) f=\mathrm{i}(g \Delta+\Delta \bar{g}), \\
& \left(2 i \omega_{\nu}+K_{+}\right) \bar{f}=\mathrm{i}(\overline{g \Delta}+\bar{\Delta} g),
\end{aligned}
$$

where we have defined the $K_{ \pm}$operators to be:

$$
\begin{aligned}
& K_{+} \bar{f}=v_{F} \hat{k} \cdot\left(2 A-\mathrm{i} \nabla_{R}\right) \bar{f}-u(\hat{k}) \cdot\left(\sigma^{\operatorname{tr}} \bar{f}+\bar{f} \sigma\right), \\
& K_{-} f=v_{F} \hat{k} \cdot\left(2 A+\mathrm{i} \nabla_{R}\right) f-u(\hat{k}) \cdot\left(\sigma f+f \sigma^{t r}\right) .
\end{aligned}
$$

The $f$ and $g$ functions denote the $\xi$-integrated Green functions:

$$
\begin{aligned}
f_{\alpha \beta}\left(\boldsymbol{R}, \hat{k} ; \mathrm{i} \omega_{\nu}\right) & =\frac{1}{\pi} \int \mathrm{d} \xi F_{\alpha \beta}\left(\boldsymbol{R}, k ; \mathrm{i} \omega_{\nu}\right), \\
i g_{\alpha \beta}\left(\boldsymbol{R}, \hat{k} ; \mathrm{i} \omega_{\nu}\right) & =\frac{1}{\pi} \int \mathrm{d} \xi G_{\alpha \beta}\left(\boldsymbol{R}, k ; \mathrm{i} \omega_{\nu}\right) .
\end{aligned}
$$

In addition, Eqs. (8) are completed with the normalization conditions:

$$
g^{2}+f \cdot \bar{f}=\sigma^{0} \text { and } \bar{g}^{2}+f \cdot \bar{f}=\sigma^{0}
$$

\section{The upper critical rotational frequency}

In this Section we assume that, as the angular velocity $\Omega$ is gradually increased, the superfluid condensate undergoes a second-order phase transition at a critical upper bound $\Omega_{\mathrm{c} 2}$. In the limit $\Omega \longrightarrow \Omega_{\mathrm{c} 2}$, therefore, the Eilenberger equations (8) can be linearized to give:

$$
\left(2 \mathrm{i} \omega_{\nu}+K_{-}\right) f=-2 \mathrm{i} \operatorname{sgn}\left(\omega_{\nu}\right) \Delta .
$$

If we apply the standard decomposition (into spin singlet and triplet parts) for the order parameter $\Delta$ and anomalous Green function $f$ :

$$
\begin{aligned}
& \Delta_{\alpha \beta}(\hat{k})=\left[\Delta_{0}(\hat{k}) \delta_{\alpha \gamma}+d(\hat{k}) \cdot \sigma_{\alpha \gamma}\right]\left(\mathrm{i} \sigma_{y}\right)_{\gamma \beta}, \\
& f_{\alpha \beta}(\boldsymbol{R}, \hat{k})=\left[f_{0}(\boldsymbol{R}, \hat{k}) \delta_{\alpha \gamma}+f(\boldsymbol{R}, \hat{k}) \cdot \sigma_{\alpha \gamma}\right]\left(\mathrm{i} \sigma_{y}\right)_{\gamma \beta},
\end{aligned}
$$

we can rewrite the linearized Gorkov equation (12) as follows:

$$
\hat{M}_{\mathrm{OP}}\left(\begin{array}{c}
f_{0} \\
f
\end{array}\right)=-2\left(\begin{array}{c}
\Delta_{0} \\
d
\end{array}\right)
$$


with

$$
\hat{M}_{\mathrm{OP}}=\left[2\left|\omega_{\nu}\right|+\operatorname{sgn}\left(\omega_{\nu}\right) v_{F} \hat{k} \cdot(\nabla-2 \mathrm{i} A)+2 \operatorname{sgn}\left(\omega_{\nu}\right)\left(\begin{array}{cc}
0 & 0 \\
0 & \hat{E}
\end{array}\right)\right],
$$

here $\hat{E}_{i j}=u_{k} \varepsilon_{i j k}$.

Let us note that the last term in Eq. (15) contains all corrections arising due to confined symmetry of the crystal and S-O interaction. From its structure one can see that for the case of spin-singlet superfluid states S-O interaction has no influence on the critical behaviour, and the equations reduce to standard BCSeigenvalue problem [9]. Therefore, we confine further our investigations to the spin-triplet states and assume the $P$-wave symmetry of order parameter (and the pairing interaction), i.e. $d_{j}(\boldsymbol{R}, \hat{k})=d_{i j}(\boldsymbol{R}) \hat{k}_{i}$ (pairing interaction constants $g_{l} \equiv 0$ for $l \neq 1)$. [10]:

By virtue of Eqs. (13)-(15) the gap equation (7) can be rewritten as follows

$$
\eta d_{i j}(R)=2 \pi T \sum_{0<\omega_{\nu}<\omega_{0}} S_{i j, m n}^{\mathrm{OP}} d_{m n}(R)
$$

where $\eta=1 / 3\left|g_{1}\right| N(0)$ and the $S^{\mathrm{OP}}$ is defined as:

$$
\begin{aligned}
& S_{i j, m n}^{\mathrm{OP}}=\int \frac{\mathrm{d} \Omega_{s}}{2 \pi} \hat{s}_{i} \hat{s}_{m} \int_{0}^{+\infty} \mathrm{d} s \exp \left[-2 s\left|\omega_{\nu}\right|\right] \exp \left[-s v_{F} \hat{\boldsymbol{s}} \cdot(\nabla-2 \mathrm{i} \boldsymbol{A})\right] \\
& \times\left[\hat{u}_{j}(\hat{s}) \hat{u}_{n}(\hat{s})+\cos (2 s u)\left(\delta_{j n}-\hat{u}_{j}(\hat{s}) \hat{u}_{n}(\hat{s})\right)\right. \\
& \left.-\sin (2 s u) \varepsilon_{j n p} \hat{u}_{p}(\hat{s})\right]
\end{aligned}
$$

where $u=|u|$ and $\hat{u}=u /|u|$.

The $d_{i j}$ tensor usually decomposed in the basis of so-called spin tensors $B_{i j}^{J M}$ (parameterized by $J, M$ quantum numbers, $J=0,1,2 ; M=-J, . ., J$; cf. [13] and also Appendix):

$$
d_{i j}(R)=\sum_{J, M} w_{J M}(R) B_{i j}^{J M}
$$

fulfills normalization condition: $d_{i j} d_{i j}^{*}=1$. Making use of orthogonality of spin tensors $B^{J M}$ (cf. Appendix) we can rewrite the gap equation (16) as (we assume $\boldsymbol{\Omega}\|\boldsymbol{v}\| \hat{\boldsymbol{\varepsilon}} \| z$-axis):

$$
\eta w_{\langle\lambda\rangle} \Phi\left(R_{x}, R_{y}\right)=2 \pi T \sum_{0<\omega_{\nu}<\omega_{c}} \tilde{S}_{\{\lambda\rangle,(\mu)}^{\mathrm{OP}} w_{\langle\mu\rangle} \Phi\left(R_{x}, R_{y}\right)
$$

where $\langle\lambda\rangle$ denotes the set of indices $\langle J M\rangle$ and 


$$
\begin{aligned}
& \tilde{S}_{\langle\lambda\rangle,\langle\mu\rangle}^{\mathrm{OP}}=S_{i j, m n}^{\mathrm{OP}} B_{i j}^{(\lambda) *} B_{m n}^{\langle\mu\rangle}=\int_{0}^{+\infty} \mathrm{d} s \exp \left(-2 s\left|\omega_{\nu}\right|\right) \\
& \times \int_{-1}^{+1} \mathrm{~d}(\cos (\vartheta)) \exp \left[2 \mathrm{i} m v_{F}|v| s \cos (\vartheta)-v_{F}^{2} m \Omega s^{2} \sin ^{2}(\vartheta)\right] \\
& \times \int_{0}^{2 \pi} \frac{\mathrm{d} \varphi}{2 \pi} \exp \left[-\mathrm{i} v_{F}(2 m \Omega)^{1 / 2} s \sin (\vartheta) \exp (\mathrm{i} \varphi) \hat{c}^{+}\right] \hat{s}_{i} \hat{s}_{m} B_{i j}^{(\lambda)^{*}} B_{m n}^{\langle\mu\rangle} \\
& \times\left[\hat{u}_{j}(\hat{s}) \hat{u}_{n}(\hat{s})+\cos (2 s u)\left(\delta_{j n}-\hat{u}_{j}(\hat{s}) \hat{u}_{n}(\hat{s})\right)-\sin (2 s u) \varepsilon_{j n p} \hat{u}_{p}(\hat{s})\right] .
\end{aligned}
$$

In the above we have introduced $\hat{c}^{+}$operator similarly as in [8-12]:

$$
\hat{c}^{+}=(8 m \Omega)^{-1 / 2}\left[-\mathrm{i}\left(\frac{\partial}{\partial R_{x}}+2 \mathrm{i} m \Omega R_{y}\right)-\frac{\partial}{\partial R_{y}}+2 \mathrm{i} m \Omega R_{x}\right],
$$

and assumed the ground-state-like function $\Phi\left(R_{x}, R_{y}\right)$ to correspond to the double periodic eigensolution constructed by Eilenberger [14], by means of which Abrikosov's vortex array solution is obtainable [15], i.e. we define it to satisfy the differential equation:

$$
\hat{c} \Phi\left(R_{x}, R_{y}\right)=0
$$

( $\hat{c}$ and $\hat{c}^{+}$operators obey the boson-type commutation relations, [12-14]).

Performing the integration with respect to $\varphi$ angle in Eq. (20) we obtain:

$$
\begin{aligned}
& \tilde{S}_{\langle\lambda\rangle,(\mu)}^{\mathrm{OP}} \Phi\left(R_{x}, R_{y}\right)=\int_{0}^{+\infty} \mathrm{d} s \exp \left(-2 s\left|\omega_{\nu}\right|\right) \\
& \times \int_{-1}^{+1} \mathrm{~d}(\cos (\vartheta)) \exp \left[2 \mathrm{i} m v_{F}|v| s \cos (\vartheta)-v_{F}^{2} m \Omega s^{2} \sin ^{2}(\vartheta)\right] \\
& \times\left[\cos (2 s \varepsilon \sin (\vartheta)) T_{\langle\lambda\rangle,\{\mu\rangle}^{(1)}+[1-\cos (2 s \varepsilon \sin (\vartheta))] T_{\langle\lambda\rangle,(\mu)}^{(2)}\right. \\
& \left.-\sin (2 s \varepsilon \sin (\vartheta)) T_{\langle\lambda\rangle,\langle\mu\rangle}^{(3)}\right) \Phi\left(R_{x}, R_{y}\right)
\end{aligned}
$$

where:

$$
\begin{aligned}
T_{\langle\lambda\rangle,\langle\mu\rangle}^{(1)} & =\left(\begin{array}{ccccc}
Z_{1} & Z_{2} & 0 & 0 & 0 \\
0 & Z_{1}^{\operatorname{tr}} & 0 & 0 & 0 \\
0 & 0 & Z_{3} & Z_{4} & 0 \\
0 & 0 & \tilde{Z}_{4} & Z_{5} & p \\
0^{\operatorname{tr}} & 0^{\operatorname{tr}} & 0^{\operatorname{tr}} & \tilde{p} & q_{1}
\end{array}\right), T_{\langle\lambda\rangle,\langle\mu\rangle}^{(2)}=\left(\begin{array}{ccccc}
Z_{6} & Z_{7} & 0 & 0 & 0 \\
0 & Z_{6}^{\operatorname{tr}} & 0 & 0 & 0 \\
0 & 0 & 0 & 0 & 0 \\
0 & 0 & 0 & Z_{8} & p \\
0^{\operatorname{tr}} & 0^{\operatorname{tr}} & 0^{\operatorname{tr}} & \tilde{p} & q_{2}
\end{array}\right), \\
T_{\langle\lambda\rangle,\langle\mu\rangle}^{(3)} & =\left(\begin{array}{ccccc}
0 & 0 & Z_{9} & Z_{10} & 0 \\
0 & 0 & 0 & Z_{11} & 0 \\
0 & Z_{9}^{\operatorname{tr}} & 0 & 0 & 0 \\
\tilde{Z}_{11} & \tilde{Z}_{10} & 0 & 0 & 0 \\
0^{\operatorname{tr}} & 0^{\operatorname{tr}} & 0^{\text {tr }} & 0^{\text {tr }} & 0
\end{array}\right)
\end{aligned}
$$

where $Z_{i}(i=1, \ldots, 11)-2 \times 2$ matrices, $p-2 \times 1$ vector and $q_{i}-$ numbers $(i=1,2)$ are defined in the Appendix. In the above 0 denotes zeroth $2 \times 2$ matrix, 
$0-2 \times 1$ zeroth vector and the operation denoted by "m" acts upon matrices : $\tilde{A}=\sigma_{x} A^{\text {tr }}$ and vectors: $\tilde{p}=\left(\sigma_{x} p\right)^{\operatorname{tr}}$. For the sake of convenience the above $T_{\langle\lambda\rangle,(\mu)}^{(\mathrm{i})}$ matrices are written in the following base: $\left[\begin{array}{ll}\mid 1 & 1\end{array}\right\rangle,|21\rangle,|1-1\rangle,|2-1\rangle,|00\rangle,|20\rangle$, $|22\rangle,|2-2\rangle,|10\rangle]$, where $|J M\rangle$ denotes the appropriate spin tensor.

We now point out that the eigenvalue problem:

$$
\begin{aligned}
& \left(\cos (2 s \varepsilon \sin (\vartheta)) T_{(\lambda\rangle,\langle\mu)}^{(1)}+[1-\cos (2 s \varepsilon \sin (\vartheta))] T_{(\lambda\rangle,(\mu)}^{(2)}\right. \\
& \left.-\sin (2 s \varepsilon \sin (\vartheta)) T_{\langle\lambda\rangle,\{\mu\rangle}^{(3)}\right) w_{(\mu)}=E w_{\{\lambda\rangle},
\end{aligned}
$$

is solved by:

$$
\begin{aligned}
& E^{(1)}=\frac{1}{2} \cos ^{2}(\vartheta)[1+\cos (2 s \varepsilon \sin (\vartheta))] \\
& w^{(1)}=2^{-1 / 2}[1,-\mathrm{i}, 0,0,0,0,0,0,0]
\end{aligned}
$$

and

$$
\begin{aligned}
& E^{(2)}=\frac{1}{4} \sin ^{2}(\vartheta)[1+\cos (2 s \varepsilon \sin (\vartheta))], \\
& w^{(2)}=[0,0,0,0,0,0,1,0,0] .
\end{aligned}
$$

Making use of this result, the eigenvalue problem (Eq. (19)) can be rewritten as:

$$
\tilde{S}_{\langle\lambda\rangle,(\mu)}^{\mathrm{OP}} w_{\langle\mu\rangle}^{(i)} \Phi\left(R_{x}, R_{y}\right)=S^{(i)}\left(\omega_{\nu}\right) \Phi\left(R_{x}, R_{y}\right) w_{\langle\mu\rangle}^{(i)} ; \quad i=1,2 ;
$$

where $S^{(i)}\left(\omega_{\nu}\right)$ denotes the eigenvalues:

$$
\begin{aligned}
& {\left[\begin{array}{l}
S^{(1)}\left(\omega_{\nu}\right) \\
S^{(2)}\left(\omega_{\nu}\right)
\end{array}\right]=\int_{0}^{+\infty} \mathrm{d} s \exp \left(-2 s\left|\omega_{\nu}\right|\right) \int_{-1}^{+1} \mathrm{~d}(\cos (\vartheta))} \\
& \times \exp \left[2 \mathrm{i} m v_{F}|v| s \cos (\vartheta)-v_{F}^{2} m \Omega s^{2} \sin ^{2}(\vartheta)\right] \\
& \times\left[\begin{array}{c}
\frac{1}{2} \cos ^{2}(\vartheta)[1+\cos (2 s \varepsilon \sin (\vartheta))] \\
\frac{1}{4} \sin ^{2}(\vartheta)[1+\cos (2 s \varepsilon \sin (\vartheta))]
\end{array}\right],
\end{aligned}
$$

of the operator in Eq: (23) corresponding to the eigenfunctions:

$$
\begin{aligned}
& d_{i j}^{(1)}\left(R_{x}, R_{y}\right)=\Phi\left(R_{x}, R_{y}\right) 2^{-1 / 2}\left[B_{i j}^{11}-\mathrm{i} B_{i j}^{21}\right], \\
& d_{i j}^{(2)}\left(R_{x}, R_{y}\right)=\Phi\left(R_{x}, R_{y}\right) B_{i j}^{22} .
\end{aligned}
$$

Let us note that since Gorkov equation (2) is invariant with respect to rotation around $\hat{\Omega}\|v\| \hat{z}$-axis, the described system conserves the $M$-quantum number (a projection of total orbital momentum). From Eq. (29) it straightforwardly follows that the resulting states obey this principle, i.e. they correspond to $M=1$ and $M=2$. The above states correspond to the appropriate solutions found in [7], i.e. the state with $M=1$ (cf. Eq. (29a)) is an axial, nonunitary solution (cf. Eq. (38,II) of [7]) and the state with $M=2$ - planar, nonunitary solution (cf. Eq. $(41, \mathrm{II})$ of 
[7]). Finally employing the definition of the critical temperature $T_{c 0}$ in the weak coupling limit we obtain (cf. [10]):

$$
\ln \left[\frac{T_{\mathrm{c} 0}}{T}\right]=2 \pi T \sum_{\nu \geq 0}\left[\frac{1}{\left|\omega_{\nu}\right|}-3 S^{(i)}\left(\omega_{\nu}\right)\right] .
$$

\section{Results of analytical and numerical calculation for $\Omega_{\mathrm{c} 2}$}

In order to solve Eq. (30) it is convenient to make use of the following dimensionless variables:

$$
t=\frac{T}{T_{\mathrm{c} 0}}, \quad \lambda v_{F}=\frac{m v_{F}^{2}}{2 \pi T_{\mathrm{c} 0}}, \quad \omega=\left[\frac{m v_{F}^{2}}{2 \pi T_{\mathrm{c} 0}}\right] \Omega_{\mathrm{c} 2}, \quad e=\frac{\varepsilon}{2 \pi T_{\mathrm{c} 0}} .
$$

Equation (30) can be transformed with accordance to techniques applied in [10-12], and finally we obtain for the state $M=1$ (cf. Eq. (29a)):

$$
\begin{aligned}
& -\ln t=\frac{3}{8} \int_{0}^{+\infty} \mathrm{d} z \exp (-z) \int_{-1}^{+1} \mathrm{~d} x\left\{\left[1-x^{2}-\left(1-3 x^{2}\right) \frac{Q}{\omega z+Q}\right]\right. \\
& \times R\left(x \frac{(\omega z+Q)^{1 / 2}}{t}\right)+\int_{0}^{2 \pi} \frac{\mathrm{d} \varphi}{2 \pi} D\left(R\left(\frac{x(\omega z+Q)^{1 / 2}-e(1-D)^{1 / 2}}{t}\right)\right. \\
& \left.\left.+R\left(\frac{x(\omega z+Q)^{1 / 2}+e(1-D)^{1 / 2}}{t}\right)\right)\right\}
\end{aligned}
$$

and for the state $M=2$ (cf. Eq. (29b)):

$$
\begin{aligned}
& -\ln t=\frac{3}{16} \int_{0}^{+\infty} \mathrm{d} z \exp (-z) \int_{-1}^{+1} \mathrm{~d} x\left\{\left[1+x^{2}+\left(1-3 x^{2}\right) \frac{Q}{\omega z+Q}\right]\right. \\
& \times R\left(x \frac{(\omega z+Q)^{1 / 2}}{t}\right)+\int_{0}^{2 \pi} \frac{\mathrm{d} \varphi}{2 \pi}(1-D)\left(R\left(\frac{x(\omega z+Q)^{1 / 2}-e(1-D)^{1 / 2}}{t}\right)\right. \\
& \left.\left.+R\left(\frac{x(\omega z+Q)^{1 / 2}+e(1-D)^{1 / 2}}{t}\right)\right)\right\}
\end{aligned}
$$

where $\left(Q=(\lambda v)^{2}\right)$ :

$$
\begin{aligned}
& D=\frac{Q}{\omega z+Q} x^{2}+\frac{\omega z}{\omega z+Q}\left(1-x^{2}\right) \cos ^{2} \varphi-2 \frac{(Q \omega z)^{1 / 2}}{\omega z+Q} x\left(1-x^{2}\right)^{1 / 2} \cos \varphi, \\
& R(y)=\operatorname{Re}\left[\psi\left(\frac{1}{2}+\mathrm{i} y\right)-\psi\left(\frac{1}{2}\right)\right] .
\end{aligned}
$$

Here $\psi$ denotes the complex digamma function (cf. [16]). Employing the well-known asymptotics of $\psi$-digamma function (cf. [16]) we obtain:

$$
R(y)= \begin{cases}\ln |y|-\psi\left(\frac{1}{2}\right)+o\left(|y|^{-1}\right) & |y| \gg 1 \\ 7 \zeta(3) y^{2}+o\left(y^{4}\right) & |y| \ll 1\end{cases}
$$


In the Ginzburg-Landau region $\left(T \sim T_{\mathrm{c}}\right)$, i.e. for $0<(1-t) \ll 1$ and $\omega \ll 1$, $Q \ll 1, e \ll 1 \mathrm{Eq} .(32)$ reduces to:

$$
\begin{aligned}
& 1-t=7 \zeta(3)\left(\frac{1}{5} \omega+\frac{2}{5} Q+\frac{1}{5} e^{2}\right) \text { for the state } M=1, \\
& 1-t=7 \zeta(3)\left(\frac{2}{5} \omega+\frac{3}{5} Q+\frac{1}{5} e^{2}\right) \quad \text { for the state } M=2,
\end{aligned}
$$

from which the slopes of the critical angular velocities $\omega$ as function of reduced temperature are easily calculated to be

$$
\frac{\mathrm{d} \omega}{\mathrm{d} t}= \begin{cases}-5 /[7 \zeta(3)] \approx-0.594 & \text { for } M=1, \\ -5 /[14 \zeta(3)] \approx-0.297 & \text { for } M=2 .\end{cases}
$$

In the limit $t=0^{+}$, on the other hand, we find from (32):

$$
\begin{aligned}
& \psi\left(\frac{1}{2}\right)=\frac{3}{8} \int_{0}^{+\infty} \mathrm{d} z \exp (-z) \int_{-1}^{+1} \mathrm{~d} x\left\{\left[1-x^{2}-\left(1-3 x^{2}\right) \frac{Q}{\omega z+Q}\right]\right. \\
& \left.\times \ln \left[x(\omega z+Q)^{1 / 2}\right]+\int_{0}^{2 \pi} \frac{\mathrm{d} \varphi}{2 \pi} D \ln \left[x^{2}(\omega z+Q)-e^{2}(1-D)\right]\right\}
\end{aligned}
$$

for $M=1$ state, and

$$
\begin{aligned}
& \psi\left(\frac{1}{2}\right)=\frac{3}{16} \int_{0}^{+\infty} \mathrm{d} z \exp (-z) \int_{-1}^{+1} \mathrm{~d} x\left\{\left[1+x^{2}+\left(1-3 x^{2}\right) \frac{Q}{\omega z+Q}\right]\right. \\
& \left.\times \ln \left[x(\omega z+Q)^{1 / 2}\right]+\int_{0}^{2 \pi} \frac{\mathrm{d} \varphi}{2 \pi}(1-D) \ln \left[x^{2}(\omega z+Q)-e^{2}(1-D)\right]\right\}
\end{aligned}
$$

for $M=2$ state.

These two equations determine the critical angular velocities $\omega_{1}$ and $\omega_{2}$ as functions of the reduced superflow $Q$ and the reduced S-O interaction factor $e$ for $T=0$. Let us note that in the limit $e=0$ (i.e. crystal is invariant with respect to spatial inversion) Eqs. (37) are the same as derived in Ref. [10]. For $Q=0$ and $e=0$ we find ( $\gamma$ is the Euler constant):

$$
\begin{aligned}
& \omega_{1}^{(0)}=\frac{1}{16} \exp \left(-\gamma+\frac{8}{3}\right) \approx 0.505, \\
& \omega_{2}^{(0)}=\frac{1}{16} \exp \left(-\gamma+\frac{5}{3}\right) \approx 0.186 .
\end{aligned}
$$

The curves in Fig. 1 were plotted from the numerical solution of Eq. (32a) and (32b) with restriction to the case of vanishing superflow for chosen values of $e$-parameter. From Fig. 1 one can see that for arbitrary strength of S-O interaction (e-parameter) the physical solution corresponds to the state $M=1$ (cf. Eq. (29a)), because in this case the critical rotational velocity is found to be the highest. Assuming in Eqs. (32) $\omega=Q=0$ we found numerically the dependence of real critical temperature $T_{\mathrm{c}}(e)$ on strength of S-O interaction. The resulting phase diagram $\mathrm{c} n$ the $(T, e)$-plane is depicted in Fig. 2. One can observe that the state $M=1$ has larger region of existence than the $M=2$ one. In Fig. 3 we depicted 

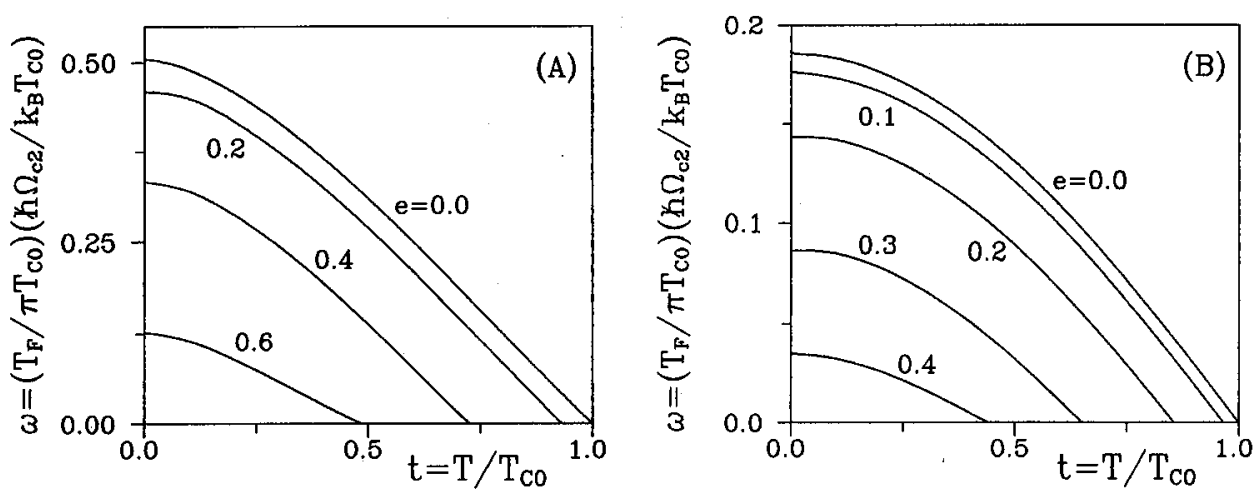

Fig. 1. Reduced critical angular velocity $\omega=\left(T_{F} / \pi T_{\mathrm{c} 0}\right)\left(\hbar \Omega_{\mathrm{c} 2} / k_{\mathrm{B}} T_{\mathrm{c} 0}\right)$ plotted versus reduced temperature $t=T / T_{\mathrm{co} 0}$ for. chosen values of $e=\varepsilon /\left(2 \pi T_{\mathrm{co}}\right)-$ strength of S-O interaction, (A) state with $M=1, e=0.0,0.2,0.4,0.6$, (B) state with $M=2, e=0.0$, $0.1,0.2,0.3,0.4$.
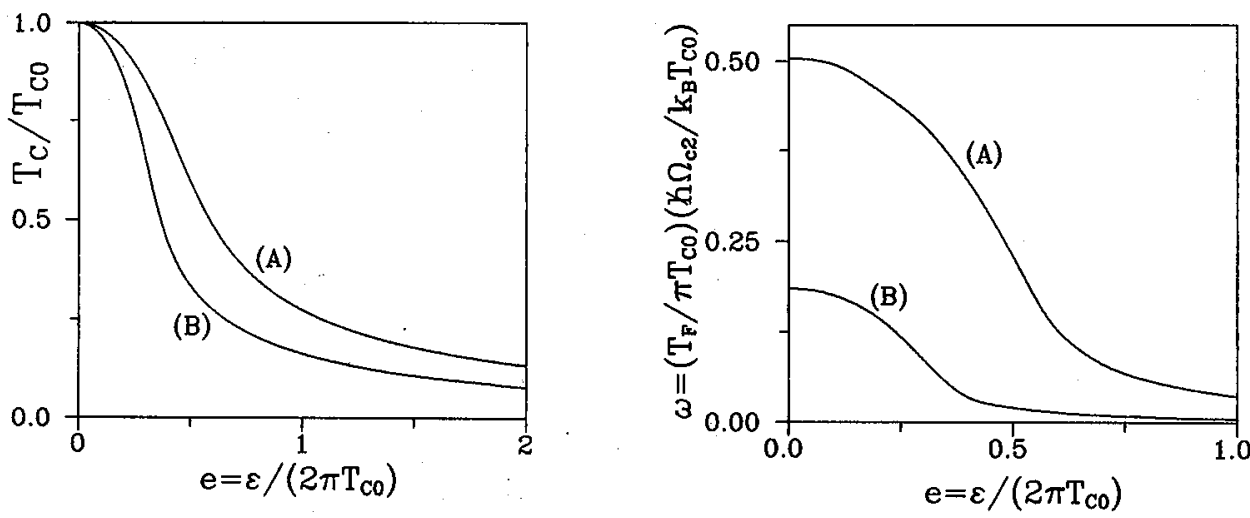

Fig. 2. Reduced critical temperature $T_{\mathrm{c}} / T_{\mathrm{co}}$ plotted versus $e=\varepsilon /\left(2 \pi T_{\mathrm{c} 0}\right)$ - strength of S-O interaction, (A) state with $M=1$, (B) state with $M=2$.

Fig. 3. Reduced critical angular velocity $\omega=\left(T_{F} / \pi T_{\mathrm{c} 0}\right)\left(\hbar \Omega_{\mathrm{c} 2} / k_{\mathrm{B}} T_{\mathrm{c} 0}\right)$ plotted versus $e=\varepsilon /\left(2 \pi T_{\mathrm{co}}\right)$ - strength of S-O interaction for zero temperature, (A) state with $M=1$, (B) state with $M=2$. 
the dependence of critical angular velocity $\omega$ on S-O interaction parameter $e$ at $T=0$. From Eqs. (37a) and (37b) the asymptotics of curves in Fig. 3 can be found analytically for $e \longrightarrow 0$ :

$$
\left\{\begin{array}{c}
\omega_{1} \approx \omega_{1}^{(0)}-\frac{2}{3}\left[\omega_{1}^{(0)} \pi\right]^{1 / 2} e^{2} \approx 0.505-0.840 e^{2} \\
\omega_{2} \approx \omega_{2}^{(0)}-\frac{2}{3}\left[\omega_{2}^{(0)} \pi\right]^{1 / 2} e^{2} \approx 0.186-0.510 e^{2}
\end{array}\right\},
$$

and for $e \longrightarrow \infty$ :

$$
\left\{\begin{array}{l}
\omega_{1} \approx\left[1 /(32 e)^{2}\right] \exp (-3 \gamma+10 / 3) \approx 4.845 \times 10^{-3} e^{-2} \\
\omega_{2} \approx\left[1 /(32 e)^{2}\right] \exp (-3 \gamma+16 / 3) \approx 3.580 \times 10^{-2} e^{-2}
\end{array}\right\} .
$$

To demonstrate the effect of superflow $v\|\Omega\| \hat{\varepsilon}$ we have also plotted in Fig. 4 the critical rotational frequencies $\omega_{1}$ and $\omega_{2}$ for $T=0$ and chosen values of $e$-parameter from a numerical solutions of Eqs. (37a) and (37b) for different values of $\lambda v$. Analytically it could be found that if $e=0$, then $\omega_{1}$ vanishes at $(\lambda v)_{1}^{(0)}=$ $\exp (-\gamma+1 / 3) / 4 \approx 0.196$, while $\omega_{2}$ does for $(\lambda v)_{2}^{(0)}=\exp (-\gamma+4 / 3) / 4 \approx 0.532$. For
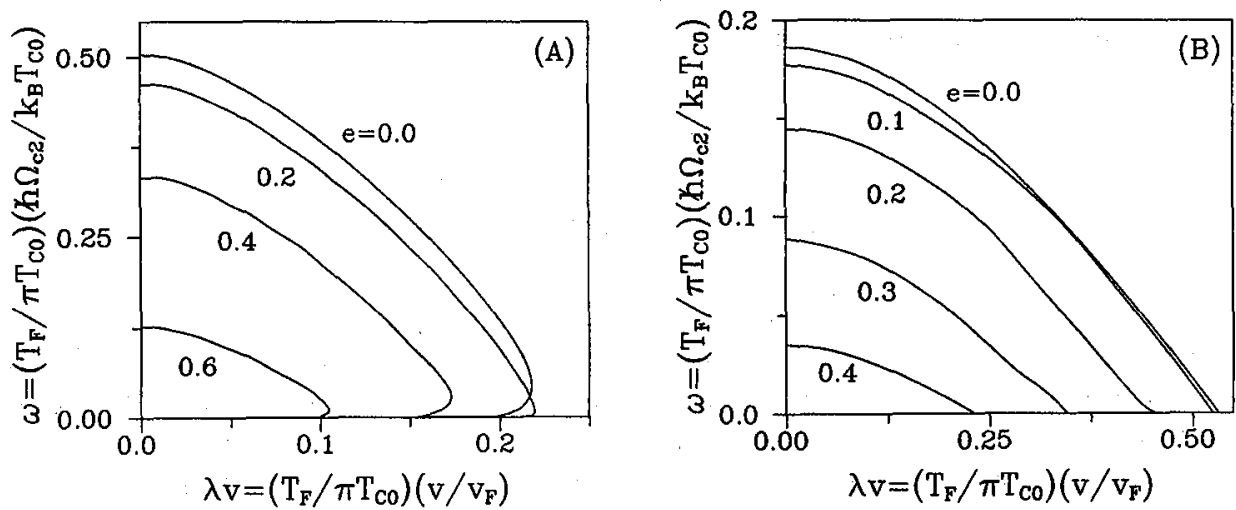

Fig. 4. Reduced critical angular velocity $\omega=\left(T_{F} / \pi T_{\mathrm{co}}\right)\left(\hbar \Omega_{\mathrm{c} 2} / k_{\mathrm{B}} T_{\mathrm{c} 0}\right)$ plotted versus reduced superflow $\lambda v=\left(T_{F} / \pi T_{\mathrm{co}}\right)\left(v / v_{F}\right)$ for zero temperature for chosen values of $e=$ $\varepsilon /\left(2 \pi T_{\mathrm{co}}\right)$ - strength of S-O interaction, (A) state with $M=1, e=0.0,0.2,0.4,0.6$, (B) state with $M=2, e=0.0,0.1,0.2,0.3,0.4$.

nonzero values of $e$-parameter, $\omega$-angular velocity vanishes at superflow velocities depicted in Fig. 5. For these we found the following asymptotics for $e \longrightarrow 0$ :

$$
\left\{\begin{array}{l}
(\lambda v)_{1} \approx(\lambda v)_{1}^{(0)}+e^{2} / 2(\lambda v)_{1}^{(0)} \approx 0.196+2.551 e^{2} \\
(\lambda v)_{2} \approx(\lambda v)_{2}^{(0)}-e^{2} /(\lambda v)_{2}^{(0)} \approx 0.532-1.880 e^{2}
\end{array}\right\},
$$

and for $e \longrightarrow \infty$ :

$$
\left\{\begin{array}{l}
(\lambda v)_{1} \approx[1 /(32 e)] \exp (--2 \gamma-5 / 3) \approx 1.861 \times 10^{-3} e^{-1} \\
(\lambda v)_{2} \approx[1 /(32 e)] \exp (-2 \gamma+13 / 6) \approx 8.600 \times 10^{-2} e^{-1}
\end{array}\right\} .
$$


Let us note that similarly to the case of $P$-wave system, without $S-O$ interaction, for some values of superflow velocity $\lambda v$, the state $M=1$ is found to be unstable against the $M=2$ one (Fig. 4, also [10]).

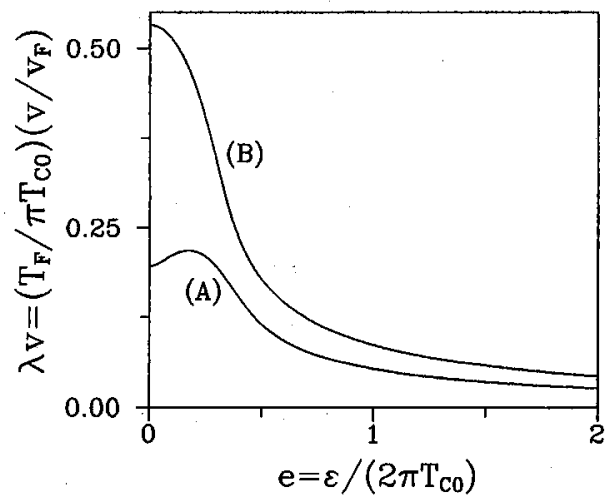

Fig. 5. Reduced superflow $\lambda v=\left(T_{F} / \pi T_{\mathrm{c} 0}\right)\left(v / v_{F}\right)$ plotted versus $e=\varepsilon /\left(2 \pi T_{\mathrm{co}}\right)-$ strength of $\mathrm{S}-\mathrm{O}$ interaction for zero temperature and vanishing rotational angular frequency.

\section{Paramagnetic critical behaviour}

In this Section we discuss paramagnetic Pauli limiting for $P$-wave pyroelectric-type superfluids. Since we are interested in critical properties only, i.e. the limit $\Delta \longrightarrow 0$, it is sufficient to discuss the linearized Eilenberger equations. Magnetic field $\boldsymbol{H}$ (acting onto spins of quasiparticles, $\boldsymbol{H} \| \boldsymbol{z}$-axis) can be easily included into Gorkov-type equations (for $P$-wave system cf. eg. [10]). At the same time Eilenberger equations (8) keep their form, while in the operators $K_{ \pm}$there appears an additional term including magnetic field:

$$
\begin{gathered}
K_{+} \bar{f}=K_{+}^{(0)} \bar{f}-H \cdot\left(\sigma^{\operatorname{tr}} \bar{f}-\bar{f} \sigma\right), \\
K_{-} f=K_{-}^{(0)} f-H \cdot\left(\sigma f-f \sigma^{\operatorname{tr}}\right),
\end{gathered}
$$

where $K_{ \pm}^{(0)}$ operators are given by Eq. (9).

We solve linearized Eilenberger equation (12) with $K_{-}$given by Eq. (43). For the sake of simplicity, in this Section we neglect the influence of superflow $v$ and rotational velocity $\Omega$ (for superconducting systems - we neglect orbital magnetic field). Applying to the order parameter the decomposition in the base of spin-tensors $B^{J M}$ (cf. Eq. (18)), after analogous operator algebra as in Section 3, we arrive at an eigenvalue problem:

$$
\eta w_{\langle\lambda\rangle}=2 \pi T \sum_{0<\omega_{\nu}\left\langle\omega_{c}\right.} \hat{S}_{\langle\lambda\rangle,\langle\mu\rangle}^{\mathrm{OP}} w_{\langle\mu\rangle}
$$


where $\hat{S}^{\mathrm{OP}}$ operator is defined as follows:

$$
\begin{aligned}
& \tilde{S}_{\langle\lambda\rangle,(\mu)}^{\mathrm{OP}}=\int_{0}^{+\infty} \mathrm{d} s \exp \left(-2 s\left|\omega_{\nu}\right|\right) \int_{-1}^{+1} \mathrm{~d}(\cos (\vartheta)) \\
& \times\left[T_{\langle\lambda\rangle,\{\mu\rangle}^{(1)} a+T_{\langle\lambda\rangle,\langle\mu\rangle}^{(2)} b-T_{\langle\lambda\rangle,\langle\mu\rangle}^{(4)} c\right],
\end{aligned}
$$

with:

$$
\begin{aligned}
& c=\frac{\varepsilon^{2} \sin ^{2}(\vartheta)}{M^{2}}[1-\cos (2 M s)], \quad a=\cos (2 M s)+c, \\
& b=\frac{H^{2}}{M^{2}}[1-\cos (2 M s)], \quad M^{2}=H^{2}+\varepsilon^{2} \sin ^{2}(\vartheta) .
\end{aligned}
$$

The $T^{(i)}(i=1,2)$ matrices in (45) are defined by (24) (we have to put $\hat{a} \equiv 0$, cf. Appendix), while $T^{(4)}$ matrix:

$$
T_{(\lambda\rangle,\langle\mu\rangle}^{(4)}=\left(\begin{array}{ccccc}
Z_{12} & 0 & 0 & 0 & 0 \\
0 & Z_{12}^{\operatorname{tr}} & 0 & 0 & 0 \\
0 & 0 & Z_{13} & 0 & 0 \\
0 & 0 & 0 & 0 & 0 \\
0^{\text {tr }} & 0^{\text {tr }} & 0^{\text {tr }} & 0^{\text {tr }} & 0
\end{array}\right),
$$

$\left(Z_{i}(i=12,13) 2 \times 2\right.$ matrices are defined in Appendix) and we have written matrices in the same base as in Eq. (23).

Similarly as in the eigenvalue problem Eq. (19), we are looking for eigenvalues (and eigenvectors) not dependent on $\omega_{\nu}$-frequencies, i.e. solve the following problem:

$$
\left(T_{\langle\lambda\rangle,\langle\mu\rangle}^{(1)} a+T_{\langle\lambda\rangle,\langle\mu\rangle}^{(2)} b-T_{\langle\lambda\rangle,\langle\mu\rangle}^{(4)} c\right) w_{\langle\mu\rangle}=E w_{(\lambda)} .
$$

Let us note that since all $T^{(i)}$ matrices in Eq. (45) are hermitean, all eigenvalues $E$ are real. We found the following eigenstates:

a) states with $M=0$ :

$$
\begin{aligned}
& E_{1}=\frac{1}{2} \sin ^{2}(\vartheta)\left\{2 \cos (2 M s)+\frac{H^{2}}{M^{2}}[1-\cos (2 M s)]\right\}, \\
& w_{1}=\left[0,0,0,0,(2 / 3)^{1 / 2},-(1 / 3)^{1 / 2}, 0,0,0\right] \\
& E_{2}=\cos ^{2}(\vartheta) \cos (2 M s) \\
& w_{2}=\left[0,0,0,0,(2 / 3)^{1 / 2},(1 / 3)^{1 / 2}, 0,0,0\right] \\
& E_{3}=\frac{1}{2} \sin ^{2}(\vartheta), w_{3}=[0,0,0,0,0,0,0,0,1] \\
& \text { b) states with } M=-2,+2
\end{aligned}
$$

$$
E_{4}=E_{5}=\frac{1}{4} \sin ^{2}(\vartheta)\left(2 \cos (2 M s)+\frac{M^{2}+H^{2}}{M^{2}}(1-\cos (2 M s))\right),
$$




$$
\begin{aligned}
& w_{4}=[0,0,0,0,0,0,1,0,0], \\
& w_{5}=[0,0,0,0,0,0,0,1,0],
\end{aligned}
$$

c) states with $M=-1,+1$ :

$$
\begin{aligned}
& E_{6}=E_{7}=\frac{1}{4} \cos ^{2}(\vartheta)\left\{2 \cos (2 M s)+\frac{M^{2}+H^{2}}{M^{2}}[1-\cos (2 M s)]\right\}, \\
& w_{6}=[1,-i, 0,0,0,0,0,0,0], \\
& w_{7}=[0,0,1, i, 0,0,0,0,0], \\
& E_{8}=E_{9}=\frac{1}{2} \sin ^{2}(\vartheta) \cos (2 M s), \\
& w_{8}=[1, i, 0,0,0,0,0,0,0], \\
& w_{9}=[0,0,1,-i, 0,0,0,0,0] .
\end{aligned}
$$

Note that states $w_{4}$ and $w_{6}$ correspond to eigenvalues of rotational velocity eigenvalue problem (Eq. (29b) and Eq. (29a), appropriately). denotes:

Finally we obtain, in the weak coupling limit, equation (30), where now $S^{(i)}$

$$
S^{(i)}\left(\omega_{\nu}\right)=\int_{0}^{+\infty} \mathrm{d} s \exp \left(-2 s\left|\omega_{\nu}\right|\right) \int_{-1}^{+1} \mathrm{~d}[\cos (\vartheta)] E_{(i)}(\vartheta),
$$

where $E_{(i)}$ are given by (49).

Making use of dimensionless variables (Eq. (31)), additionally assuming:

$$
h=\frac{H}{2 \pi T_{\mathrm{c} 0}},
$$

and applying the techniques from Section 4 we finally arrive at the following implicit equations determining the Pauli limiting paramagnetic critical field $h$ :

$$
\begin{aligned}
& -\ln t=\frac{3}{4} \int_{-1}^{+1} \mathrm{~d} x\left(1-x^{2}\right)^{2} \frac{e^{2}}{h^{2}+e^{2}\left(1-x^{2}\right)} R\left(\frac{N}{t}\right), \\
& -\ln t=\frac{3}{2} \int_{-1}^{+1} \mathrm{~d} x x^{2} R\left(\frac{N}{t}\right),
\end{aligned}
$$

$\ln t \equiv 0$,

$$
\begin{aligned}
& -\ln t=\frac{3}{8} \int_{-1}^{+1} \mathrm{~d} x\left(1-x^{2}\right)^{2} \frac{e^{2}}{h^{2}+e^{2}\left(1-x^{2}\right)} R\left(\frac{N}{t}\right), \\
& -\ln t=\frac{3}{4} \int_{-1}^{+1} \mathrm{~d} x\left(1-x^{2}\right) x^{2} \frac{e^{2}}{h^{2}+e^{2}\left(1-x^{2}\right)} R\left(\frac{N}{t}\right),
\end{aligned}
$$




$$
-\ln t=\frac{3}{4} \int_{-1}^{+1} \mathrm{~d} x\left(1-x^{2}\right) R\left(\frac{N}{t}\right),
$$

where $N=\left(e^{2}\left(1-x^{2}\right)+h^{2}\right)^{1 / 2}$. The above equations appropriately correspond to the states in (49).

Let us figure out that from identity (52c) it follows that the state $(49 \mathrm{c})$ is neither influenced by magnetic field, nor by the $\mathrm{S}-\mathrm{O}$ interaction (at least in the weak coupling limit). We note that if $e=0$ states (49a), (49d) and (49e) have no Pauli limiting magnetic field also. Moreover from numerical solutions of Eqs. (52), presented in Figs. 6, 7 and 8, it follows that three distinct types of phase diagrams (on ( $\mathrm{T}, \mathrm{H})$-plane) realize.

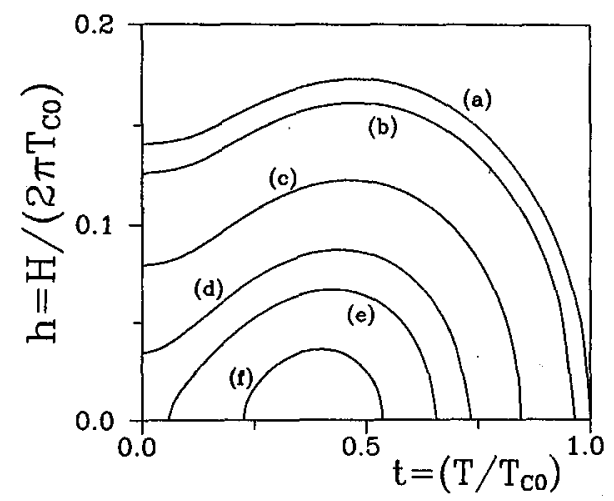

Fig. 6. Phase diagram reduced magnetic field $h=H /\left(2 \pi T_{\mathrm{co}}\right)$ versus reduced temperature $t=T / T_{\mathrm{co}}$ for chosen values of $e=\varepsilon /\left(2 \pi T_{\mathrm{co}}\right)$ - strength of S-O interaction for the $M=0$ state defined in Eq. (49b), (a) $e=0.0$, (b) $e=0.1$, (c) $e=0.2$, (d) $e=0.25$, (e) $e=0.27$, (f) $e=0.29$.

Let us briefly discuss the main features of obtained phase diagrams. The first type of the phase diagram (Fig. 6) corresponds to the state (49b) (and also state (49f) has similar phase diagram). In this case we deal with Pauli limiting for arbitrary value of S-O interaction $e$-parameter. For $e=0$ we find analytically $h=\exp (-\gamma) / 4 \approx 0.140$ at $T=0$. For $0 \leq e \leq \exp (-\gamma+4 / 3) / 8 \approx 0.266$ the region of existence of this state is enclosed between $t$-, $h$-axis and the critical curve (see curves (a)-(d) in Fig. 6). For $0.266 \leq e \leq 0.298$ the region of existence of superfluid state $(49 \mathrm{~b})$ is enclosed between $t$-axis and critical curve (see curves (e)-(f) in Fig. 6). We note that in this regime the system at low temperatures $(T \approx 0)$ is normal. Gradually increasing temperature the system undergoes the phase transition to the superfluid phase, and finally reenters the normal phase. For stronger S-O interaction, i.e. $e \geq 0.298$ the superfluid state is not stable against normal one.

The critical paramagnetic behaviour of solutions of Eq. (52a) is depicted in Fig. 7. Here for $e=0$ we have no Pauli limiting, and for $H=0$ the superfluid state realizes for $e \leq 0.196$. For $0 \leq e \leq \exp (-\gamma+5 / 6) / 8 \approx 0.161$, the region of existence 

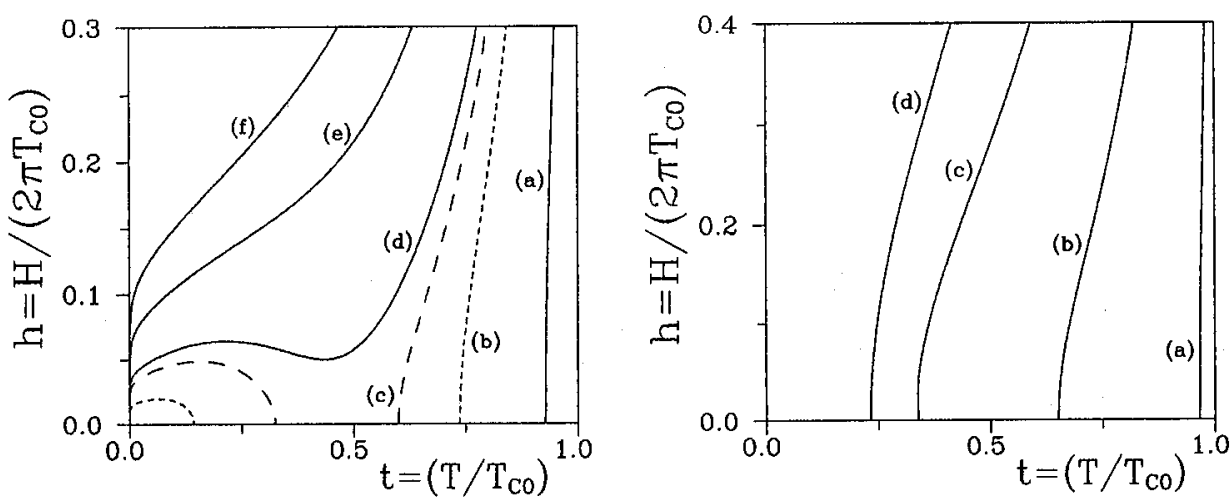

Fig. 7. Phase diagram reduced magnetic field $h=H /\left(2 \pi T_{\mathrm{co}}\right)$ versus reduced temperature $t=T / T_{\mathrm{co} 0}$ for chosen values of $e=\varepsilon /\left(2 \pi T_{\mathrm{c} 0}\right)$ - strength of S-O interaction for the $M=0$ state defined in Eq. (49a), (a) $e=0.1$, (b) $e=0.17$ (dashed line), (c) $e=0.19$ (dashed line), (d) $e=0.2$, (e) $e=0.25$, (f) $e=0.3$.

Fig. 8. Phase diagram reduced magnetic field $h=H /\left(2 \pi T_{\mathrm{co}}\right)$ versus reduced temperature $t=T / T_{\mathrm{c} 0}$ for chosen values of $e=\varepsilon /\left(2 \pi T_{\mathrm{c} 0}\right)-$ strength of S-O interaction for the $M=2$ state defined in Eq. (49d), (a) $e=0.1$, (b) $e=0.3$, (c) $e=0.5$, (d) $e=0.7$.

of the superfluid state is infinite (in the weak coupling limit) and is delimited by $t$-, h-axis and the critical curve (see curve (a) in Fig. 7). For $0.161 \leq e \leq 0.196$ the pairing condensate exists also in infinite region delimited by $t-, h$-axis and two branches of the critical curve. Note that the region between the $t$-, $h$-axis and left branch of critical curve (see curves (b)-(c) in Fig. 7) corresponds to the normal state. Finally for $e \geq 0.196$ the region of existence of state (49a) lies above the critical curve (see curves (d)-(f) in Fig. 7). In this regime, however, for low magnitudes of magnetic field the state appears to be normal, while increasing field the system undergoes a phase transition to the superfluid state. Note that when $h \longrightarrow 0$ all critical curves tend asymptotically to line $t=1$.

The remaining states (49d) and (49e) (Fig. 8 corresponds to solutions of Eq. (52d)) have the superfluid solution for arbitrary regime of $e$ and $h$ parameters. The region of existence of superfluid phase is infinite and delimited by $t$-, $h$-axis and critical curve (see curves (a)-(d) in Fig. 8).

\section{Appendix}

Assuming that the projection of angular momentum of the system is quantized with respect to $z$-axis, the spherical tensors, $B_{i j}^{J m}=|J m\rangle_{i j}$, applied for expansion of $P$-wave order parameter (cf. Eq. (18)) are defined as:

$$
|00\rangle_{i j}=3^{-1 / 2} \delta_{i j},|10\rangle_{i j}=2^{-1 / 2}\left(\delta_{i 2} \delta_{j 1}-\delta_{i 1} \delta_{j 2}\right),
$$




$$
\begin{aligned}
& |11\rangle_{i j}=\frac{1}{2}\left(\delta_{i 2} \delta_{j 3}-\delta_{i 3} \delta_{j 2}+i \delta_{i 3} \delta_{j 1}-i \delta_{i 1} \delta_{j 3}\right), \\
& |20\rangle_{i j}=-6^{-1 / 2}\left(\delta_{i j}-3 \delta_{i 3} \delta_{j 3}\right), \\
& |21\rangle_{i j}=-\frac{1}{2}\left(\delta_{i 1} \delta_{j 3}+\delta_{i 3} \delta_{j 1}+i \delta_{i 2} \delta_{j 3}+i \delta_{i 3} \delta_{j 2}\right), \\
& |22\rangle_{i j}=\frac{1}{2}\left(\delta_{i 1} \delta_{j 1}-\delta_{i 2} \delta_{j 2}+i \delta_{i 1} \delta_{j 2}+i \delta_{i 2} \delta_{j 1}\right) .
\end{aligned}
$$

The tensors $\mid J-m)_{i j}$ are defined as

$$
|J-m\rangle_{i j}=(-1)^{m}|J m\rangle_{i j}^{*} \text {. }
$$

The spin tensors obey the following orthogonalization conditions:

$$
\begin{aligned}
& \left|J_{1} m_{1}\right\rangle_{i j}\left|J_{2} m_{2}\right\rangle_{i j}^{*}=\delta_{J_{1} J_{2}} \delta_{m_{1} m_{2}} \\
& |J m\rangle_{i j}|J m\rangle_{k n}^{*}=\delta_{i k} \delta_{j n} .
\end{aligned}
$$

The $Z_{i}(i=1, \ldots, 13)-2 \times 2$ matrices, $p-2 \times 1$ vector and $q_{i}-$ numbers $(i=1,2)$ used in Eq. (24) are defined as follows:

$$
\begin{aligned}
& Z_{1}=\frac{1}{4}\left(\begin{array}{cc}
\left(1+\cos ^{2}(\vartheta)\right) & -\mathrm{i}\left(1-3 \cos ^{2}(\vartheta)\right) \\
\mathrm{i}\left(1-3 \cos ^{2}(\vartheta)\right) & \left(1+\cos ^{2}(\vartheta)\right)
\end{array}\right) \\
& Z_{2}=\frac{1}{8} \sin ^{2}(\vartheta) \hat{a}^{2}\left(\begin{array}{cc}
1 & i \\
i & -1
\end{array}\right), \quad Z_{4}=\frac{1}{4} \sin ^{2}(\vartheta) \hat{a}^{2}\left(\begin{array}{cc}
0 & 1 \\
0 & -6^{1 / 2}
\end{array}\right) \\
& Z_{3}=\frac{1}{3}\left(\begin{array}{cc}
1 & -2^{-1 / 2}\left(1-3 \cos ^{2}(\vartheta)\right) \\
-2^{-1 / 2}\left(1-3 \cos ^{2}(\vartheta)\right) & \frac{1}{2}\left(1+3 \cos ^{2}(\vartheta)\right)
\end{array}\right) \\
& Z_{5}=\frac{1}{2}\left(1-\cos ^{2}(\vartheta)\right)\left(\begin{array}{ll}
1 & 0 \\
0 & 1
\end{array}\right), \quad Z_{6}=\frac{1}{4} \cos ^{2}(\vartheta)\left(\begin{array}{cc}
1 & i \\
-i & 1
\end{array}\right) \\
& Z_{7}=\frac{1}{8} \cos ^{2}(\vartheta) \hat{a}^{2}\left(\begin{array}{cc}
-1 & i \\
i & 1
\end{array}\right), \quad Z_{8}=\frac{1}{4} \sin ^{2}(\vartheta)\left(\begin{array}{cc}
1 & -\hat{a}^{4} / 6 \\
0 & 1
\end{array}\right) \\
& Z_{9}=\frac{i \hat{a}}{2 \cdot 3^{1 / 2}}\left(\begin{array}{cc}
1 & -2^{-1 / 2}\left(1-3 \cos ^{2}(\vartheta)\right) \\
-\left(1-2 \cos ^{2}(\vartheta)\right) & 2^{1 / 2}\left(1+\cos ^{2}(\vartheta)\right)
\end{array}\right), \\
& Z_{10}=\frac{1}{24} \sin ^{2}(\vartheta) \hat{a}^{3}\left(\begin{array}{cc}
0 & i \\
0 & -1
\end{array}\right), \quad Z_{11}=\frac{1}{4} \sin ^{2}(\vartheta) \hat{a}\left(\begin{array}{ll}
0 & i \\
0 & 1
\end{array}\right), \\
& Z_{12}=\frac{1}{4} \sin ^{2}(\vartheta)\left(\begin{array}{cc}
1 & -i \\
i & 1
\end{array}\right), \quad Z_{13}=\frac{1}{3} \cos ^{2}(\vartheta)\left(\begin{array}{cc}
1 & 2^{1 / 2} \\
2^{1 / 2} & 2
\end{array}\right)
\end{aligned}
$$




$$
\begin{aligned}
& p=\frac{i}{4 \cdot 2^{1 / 2}} \sin ^{2}(\vartheta) \hat{a}^{2}\left(\begin{array}{l}
1 \\
0
\end{array}\right), \quad q_{1}=\frac{1}{2}\left(1-\cos ^{2}(\vartheta)\right), \\
& q_{2}=\frac{1}{4} \sin ^{2}(\vartheta),
\end{aligned}
$$

where $\hat{a}=-\mathrm{i} v_{F}(2 m \Omega)^{1 / 2} s \sin (\vartheta) \hat{c}^{+}$.

\section{References}

[1] L.N. Bulaevskii, A.I. Rusinov, Pisma Zh. Eksp. Teor. Fiz. 21, 147 (1975).

[2] L.N. Bulaevskii, A.A. Gusieinov, A.I. Rusinov, Zh. Eksp. Teor. Fiz. 21, 2356 (1976).

[3] A.A. Abrikosov, L.P. Gorkov, Zh. Eksp. Teor. Fiz. 42, 1088 (1962).

[4] N.R. Werthamer, E. Helfand, P.C. Hohenberg, Phys. Rev. 147, 295 (1966).

[5] J.A. Wilson, F.J. DiSalvo, S. Mahagan, Adv. Phys. 24, 117 (1975).

[6] G.S. Parry, C.B. Seruby, P.W. Williams, Philos. Mag. 29, 601 (1974).

[7] I.H. Krzyżanowski, Acta Phys. Pol. A78, 885 (1990).

[8] G. Eilenberger, Z. Phys. 214, 195 (1968).

[9] E. Helfand, N.R. Werthamer, Phys. Rev. 147, 288 (1966).

[10] N. Schopohl, J. Low Temp. Phys. 41, 409 (1980).

[11] H. Monien, K. Scharnberg, L. Tewordt, N. Schopohl, J. Low Temp. Phys. 65, 13 (1986).

[12] I.H. Krzyżanowski, Acta Phys. Pol. A78, 899 (1990).

[13] E. ElBaz, B. Castel, Graphical Methods of Spin Algebras, Marcel Dekker, Inc., New York 1972.

[14] G. Eilenberger, Phys. Rev. 153, 584 (1967).

[15] A.L. Fetter, P.C. Hohenberg, in Superconductivity, ed. R.D. Parks, Marcel Dekker, Inc., New York 1969.

[16] M. Abramowitz, I.A. Stegun, Handbook of Mathematical Functions, Dover, New York 1970. 